\title{
Inhibition of RAB1A suppresses epithelial-mesenchymal transition and proliferation of triple-negative breast cancer cells
}

\author{
HUI XU*, MINGPING QIAN*, BINGKUN ZHAO, CHENYANG WU, NIRAJ MASKEY, \\ HONGMING SONG, DENGFENG LI, JIALU SONG, KAIYAO HUA and LIN FANG \\ Department of Breast and Thyroid Surgery, Shanghai Tenth People's Hospital, School of Medicine, \\ Tongji University, Shanghai 200072, P.R. China
}

Received August 26, 2016; Accepted January 16, 2017

DOI: $10.3892 /$ or.2017.5404

\begin{abstract}
RAB1A acts as an oncogene in various cancers, and emerging evidence has verified that RAB1A is an mTORC1 activator in hepatocellular and colorectal cancer, but the role of RAB1A in breast cancer remains unclear. In this investigation, RAB1A siRNA was successfully transfected in MDA-MB-231 and BT-549 human triple-negative breast cancer cells, and verified by real-time quantitative polymerase chain reaction and western blotting. Then, MTT cell proliferation, colony formation, cell invasion and wound healing assays were performed to characterize the function of RAB1A in the breast cancer cell lines. Downregulation of RAB1A inhibited cellular growth, cell migration, cell invasion and cell epithelial-mesenchymal transition. Furthermore, compared with NC siRNA transfected cells, RAB1A siRNA transfected breast cancer cells inhibited the phosphorylation of $\mathrm{S} 6 \mathrm{~K} 1$, the effector molecular of mTORC1. Collectively, our data suggested that RAB1A acts as an oncogene by regulating cellular proliferation, growth, invasion and metastasis via activation of mTORC1 pathway in triple-negative breast cancer.
\end{abstract}

Correspondence to: Professor Lin Fang or Hui Xu, Department of Breast and Thyroid Surgery, Shanghai Tenth People's Hospital, School of Medicine, Tongji University, 301 Yanchang Road, Zhabei, Shanghai 200072, P.R. China

E-mail: syjrkfanglin@126.com

E-mail: 18516336044@163.com

*Contributed equally

Abbreviations: EMT, epithelial-mesenchymal transition; RT-qPCR, real-time quantitative polymerase chain reaction; DMSO, dimethyl sulfoxide; MTT, 3-(4,5-dimethylthiazoyl-2-yl)-2,5-diphenyl tetrazolium bromide

Key words: RAB1A, triple-negative breast cancer, mTORC1, epithelialmesenchymal transition

\section{Introduction}

According to the investigation conducted in 2015, approximately 429,000 new cancers cases and 281,4000 cancer deaths would occur in China (1). Breast cancer ranks as the first leading cause of cancer-related death among women in less developed countries (2,3). For most breast cancer patients, surgical removal of the tumor is the first step of treatment, adjuvant therapies are recommended to follow, including systemic treatment with chemotherapy, endocrine therapy, targeted therapy and postoperative radiation therapy. Substantial advances have been made in the prognosis and treatment of breast cancers. However, the treatment progress and prevention effects were minimal. The need towards deeper understanding the pathogenesis of breast cancer is highlighted and will advance the development of novel strategies for effective control of this disease.

Member of Rab family (4), RAB1A is anchored on the membranes of endoplasmic reticulum (ER) and Golgi by prenylation and functions as controller of vesicle trafficking from ER to Golgi apparatus $(5,6)$. The aberrant expression of RAB1A induces many diseases, such as Parkinson's disease (7), aspirin-exacerbated respiratory disease (8), cardiomyopathy (9) and cancer $(10,11)$. mTORC1 as a complicated pathway is demonstrated to play various effects on cell survival, cell growth, cell metabolism, cell cycle and is sensitive to rapamycin (12). Mounting studies conducted recently found RAB1A is involved in the regulation of $\mathrm{mTORC} 1$ signaling, in colorectal, prostate and hepatocellular cancer (13-15). In 2014, Thomas et al revealed that Rab1A is an mTORC1 activator, and can stimulate oncogenic growth via mTORC1 pathway with the presence of amino acid (AA) in colorectal cancer (13). Gulhati et al reported a study on the effect of mTORC1 in regulating epithelial-mesenchymal transition (EMT) (16).

To our knowledge, characterization of RAB1A is less well developed. Thus, one goal of this study was to determine the function of RAB1A in breast cancer and its relationship with mTOR pathway. Our results may have an effect on the treatment and prognosis of breast cancer.

\section{Materials and methods}

Cell culture. The breast cancer cell lines MDA-MB231 and BT-549 were purchased from the Cell Bank of 
the Chinese Academy of Sciences (Shanghai, China). MDA-MB-231 cells were cultured in Dulbecco's modified Eagle's medium (DMEM) supplemented with $10 \%$ fetal bovine serum (FBS) (Gibco, Carlsbad, CA, USA), penicillin (100 U/ $\mathrm{ml})$ and streptomycin $(100 \mu \mathrm{g} / \mathrm{ml})$ (Enpromise, Hangzhou, China). BT-549 cells were grown in RPMI-1640 medium supplemented with 10\% FBS (Gibco), penicillin (100 U/ml) and streptomycin $(100 \mu \mathrm{g} / \mathrm{ml})$ (both from Enpromise). The cells were incubated at $37^{\circ} \mathrm{C}$ in a humidified atmosphere containing $5 \% \mathrm{CO}_{2}$.

RAB1A small interfering RNA (siRNA) and negative control siRNA (NC siRNA) oligonucleotides were synthesized by GenePharma (Shanghai, China). The sequence of RAB1A siRNAs was sense, 5'-CAGCAUGAAUCCCGAAUAUTT-3' and antisense, 5'-AUAUUCGGGAUUCAUGCUGTT-3'; the sequence of NC siRNA was sense, 5'-UUCUCCGAACGUGU CACGUTT-3' and antisense, 5'-ACGUGACACGUUCGGA GAATT-3'.

Cell transfection. For transfection, MDA-MB-231 and BT-549 cells were seeded into 6-well plates with a starting cell number of $12 \times 10^{4}$ and cultured with serum and antibiotic free DMEM or RPMI-1640 medium, respectively. Cells were transfected using Lipofectamine 2000 transfection reagent (Invitrogen Life Technologies, Carlsbad, CA, USA) according to the instructions provided by the manufacturer when cells density reached $50-60 \%$. The medium was replaced by complete DMEM or RPMI-1640 medium after $6 \mathrm{~h}$ of incubation. The cells were used for future analysis after $48 \mathrm{~h}$ transfection.

$R N A$ isolation, reverse transcription and real-time quantitative polymerase chain reaction ( $R T-q P C R)$. TRIzol reagent (Invitrogen Life Technologies) was used to isolate total RNA according to the manufacturer's instructions after $48 \mathrm{~h}$ transfection. RNA was reverse-transcribed with PrimeScript RT-PCR kit (Takara Bio Inc., Tokyo, Japan), according to the manufacturer's instructions. Conditions of the reverse transcription (RT) reaction were $37^{\circ} \mathrm{C}$ for $15 \mathrm{~min}$, then $85^{\circ} \mathrm{C}$ for $5 \mathrm{sec}$. The SYBR-Green PCR master mix (Takara Bio Inc.) was used for RT-qPCR, which was followed by detection with a 7900HT fast RT-PCR instrument (Applied Biosystems, Singapore). GAPDH was used as an internal standard.

RAB1A mRNA expression was assessed with the following primers: 5'-TTGCCTTCTTCTTAGGTTTGC-3' (forward), and 5'-GCTTGATTGTTTTCCCGTCT-3' (reverse). RT-qPCR parameters for quantification were as follows: $2 \mathrm{~min}$ at $95^{\circ} \mathrm{C}$, followed by 40 cycles of $15 \mathrm{sec}$ at $95^{\circ} \mathrm{C}$ and $30 \mathrm{sec}$ at $60^{\circ} \mathrm{C}$. The relative expression was calculated using the relative quantification equation $(R Q)=2^{-\Delta \Delta C t}$. Each sample was performed in triplicate.

Cell proliferation assay. Following $24 \mathrm{~h}$ transfection, the cells were seeded at $2 \times 10^{3}$ cells/well in 96-well plates. Cell growth was monitored every day for a period of 5 days. Then $20 \mu \mathrm{l}$ of 3-(4,5-dimethylthiazol-2-yl)-2,5-diphenyltetrazolium bromide (MTT; Sigma-Aldrich, St. Louis, MO, USA) solution was added in each well and further incubated for $4 \mathrm{~h}$. Then $150 \mu$ ldimethyl sulfoxide (DMSO; Sigma-Aldrich) was added in each well and shaken for 10 min gently to dissolve the MTT formazan crystals after removing the supernatant. Absorbance was recorded at $490 \mathrm{~nm}$ with a microplate reader (BioTek Instruments, Inc., Winooski, VT, USA).

Plate colony formation assay. MDA-MB-231 and BT-549 cells were seeded in a 6 -well plate at 500 cells/well after $24 \mathrm{~h}$ of transfection, incubated for 1 week at $37^{\circ} \mathrm{C}$ in humidified $5 \% \mathrm{CO}_{2}$ conditions. Cells were washed with PBS to remove the debris and fixed by $95 \%$ ethanol for $10 \mathrm{~min}$, dried and stained with $0.1 \%$ crystal violet solution for $20 \mathrm{~min}$. The number of colonies with diameters of more than $1.5 \mathrm{~mm}$ was counted after washing with tap water 3 times.

Cell invasion assay. Transwell chambers (Corning Inc., Lowell, MA, USA) with a pore size of $8 \mu \mathrm{m}$ were used for invasion assay and were pre-coated with Matrigel. Cells were harvested after transfected by RAB1A siRNA or NC siRNA and resuspended with DMEM or RPMI-1640 medium without FBS. Medium $(200 \mu \mathrm{l})$ containing $5 \times 10^{4}$ cells were added into the upper chamber with $0.1 \%$ BSA solution and added to the 24-well plate. Complete DMEM or RPMI-1640 medium was added into the bottom chamber, serving as a chemoattractant. After $16 \mathrm{~h}$ incubation at $37^{\circ} \mathrm{C}$ in $5 \% \mathrm{CO}_{2}$, cells on the upper surface were carefully removed with a cotton swab. Cells penetrated to the lower surface of the membrane were fixed with $10 \%$ formalin, stained with crystal violet and counted under a microscope. Results from 1 of 3 representative experiments are shown.

Wound healing assay. To evaluate the cell mobility, wound healing assay was conducted. MDA-MB-231 and BT-549 cells were transfect. Six-well plates were used with $15 \times 10^{4}$ cells/ well. The plates were washed 3 times with PBS, when the confluence of cells reached $\sim 90 \%$, a scratch was made using a sterile pipette tip. The process of wound healing was observed at $0,12,24$ and $48 \mathrm{~h}$ after incubating at $37^{\circ} \mathrm{C}$ in $5 \% \mathrm{CO}_{2}$. Representative migration images are presented. Each treatment was performed in triplicate.

Protein extraction and western blotting. Total cell protein content was extracted after $48-72 \mathrm{~h}$ transfection by using radio immunoprecipitation assay (RIPA) lysis buffer ( $80 \mu \mathrm{l} /$ well; Beyotime Institute of Biotechnology, Jiangsu, China). The supernatants were collected and centrifuged at $4^{\circ} \mathrm{C}$, then protein concentrations were qualified by BCA protein assay kit (Beyotime Institute of Biotechnology). Subsequently, protein samples were denatured with $6 \mathrm{X}$ sodium dodecyl sulfate (SDS) loading buffer at $95^{\circ} \mathrm{C}$ for $5 \mathrm{~min}$. Protein lysates were resolved by 10 or $12 \%$ SDS-polyacrylamide gel electrophoresis (SDS-PAGE), then transferred onto a $0.45 \mu \mathrm{m}$ nitrocellulose membrane (both from Beyotime Institute of Biotechnology). Later, the membranes were blocked with 5\% skim milk for $1 \mathrm{~h}$ and then incubated overnight at $4^{\circ} \mathrm{C}$ with primary polyclonal or monoclonal antibodies as follows: anti-RAB1A (rabbit, 1:500; Proteintech, Chicago, IL, USA), anti- $\beta$-actin (mouse, 1:1,000), anti-E-cadherin (mouse, 1:750), anti-N-cadherin (mouse, 1:750), anti-vimentin (mouse, 1:750), anti-ERK (mouse, 1:1,000), anti-phospho-ERK (mouse, 1:1,000), anti-AKT (mouse, 1:1,000), anti-phospho-AKT (mouse, 1:1,000) and anti-pS6K (mouse, 1:1,000) (all from Cell Signaling Technology, Inc., Danvers, 
A

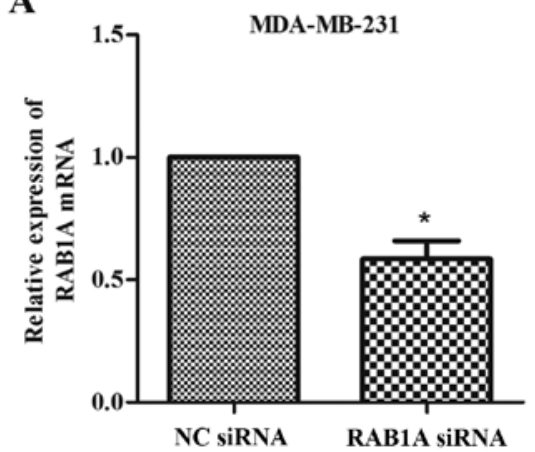

B

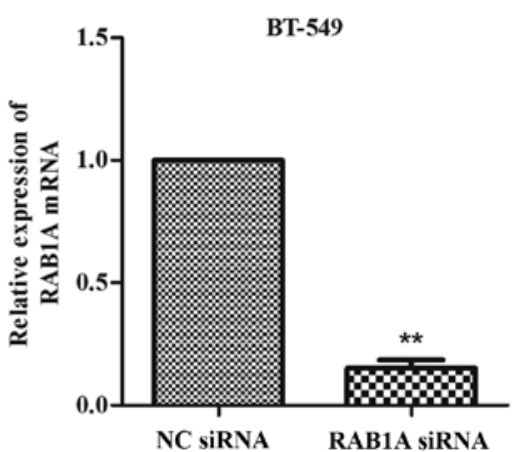

Figure 1. Relative mRNA expression of RAB1A is downregulated by RAB1A siRNA in MDA-MB-231 and BT-549 cells. RAB1A mRNA expression as determined by real-time PCR is presented. Columns show means \pm SEM. (A) MDA-MB-231 cells, ${ }^{*} \mathrm{P}<0.05$ vs. the control by two-tailed t-test. (B) BT-549 cells, ${ }^{* *} \mathrm{P}<0.01$ vs. the control by two-tailed t-test. $\mathrm{PCR}$, polymerase chain reaction.

A

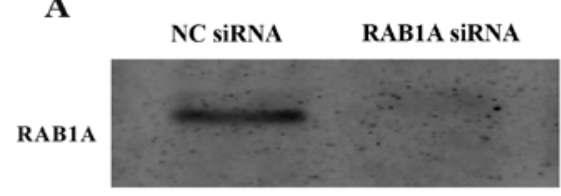

ACTIN

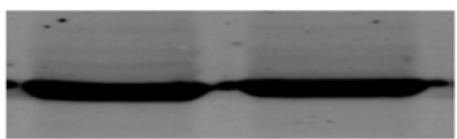

C

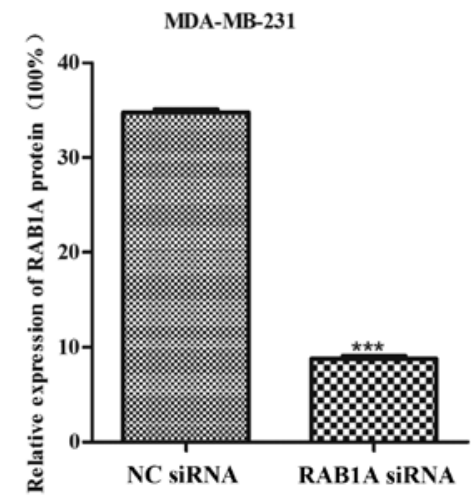

B
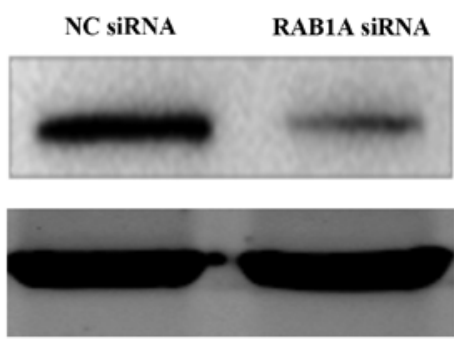

D

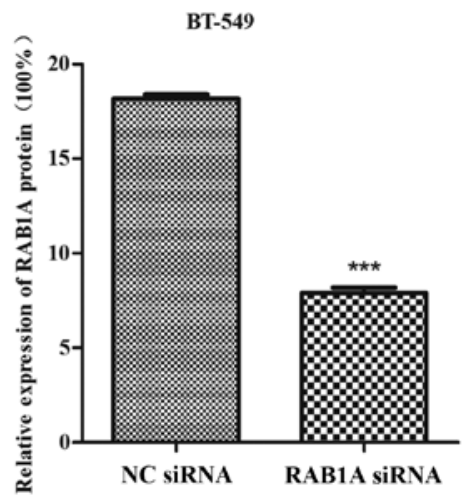

Figure 2. (A and B) Expression of RAB1A protein is inhibited in RAB1A siRNA transfected breast cancer cells. RAB1A protein levels in (C) MDA-MB-231 and (D) in BT-549 cells were lower than NC when induced by RAB1A siRNA. $\beta$-actin served as the loading control. The graph shows the mean \pm SEM of $\mathrm{RAB1A}$ protein levels relative to their respective loading controls. Results are representative of two similar experiments. NC, negative control. ${ }^{* * *} \mathrm{P}<0.001$.

MA, USA), anti-phospho-pS6K (Ser-418, mouse, 1:1,000; Ruiying Biological, Jiangsu, China). Next, the membranes were washed three times with PBST for $10 \mathrm{~min}$ each time, and incubated with anti-mouse or anti-rabbit secondary antibody (1:1,000; Epitomics, Burlingame, CA, USA) for $1 \mathrm{~h}$ at room temperature. Finally, after 3 times wash with PBST, the target proteins were detected with an Odyssey Scanning system (LI-COR Biosciences, Lincoln, NE, USA). The expression levels of the target protein were normalized to those of $\beta$-actin or tubulin. Each treatment was performed in triplicate.

Statistical analysis. The data were analyzed using the SPSS, version 20.0 (IBM Corp., Somers, NY, USA) or GraphPad Prism, version 6.0 (GraphPad Software, San Diego, CA, USA). The data were expressed as the mean \pm standard error of the mean (SEM) for at least 3 repeated individual experiments for each group. Statistical analyses were performed using an unpaired two-tailed Student's t-test. P-values $<0.05$ were considered statistically significant.

\section{Results}

Expression of RABIA is downregulated by SiRABIA in breast cancer cells. The RT-qPCR and western blotting were conducted to analyze the expression of RAB1A at the protein level and mRNA level, respectively. As shown in Fig. 1A and B, MDA-MB-231 and BT-549 cells were successfully transfected with siRAB1A, leading to the significant suppression of RAB1A mRNA expression. Furthermore, the expression of target proteins were effectively inhibited in siRNA transfected breast cancer cells compared with NC cells (Fig. 2; $\mathrm{P}<0.001$ ). According to the experiments above, the suppression effects of siRAB1A on RAB1A expression were verified. 
A
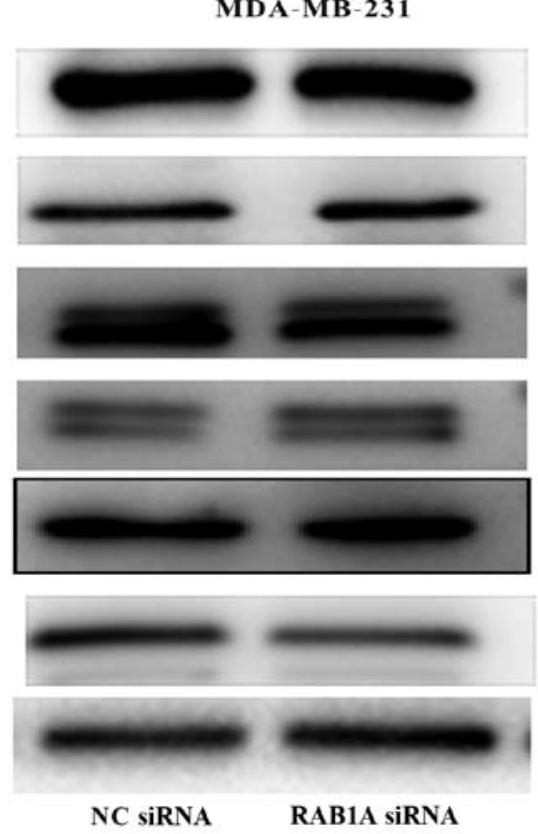

C

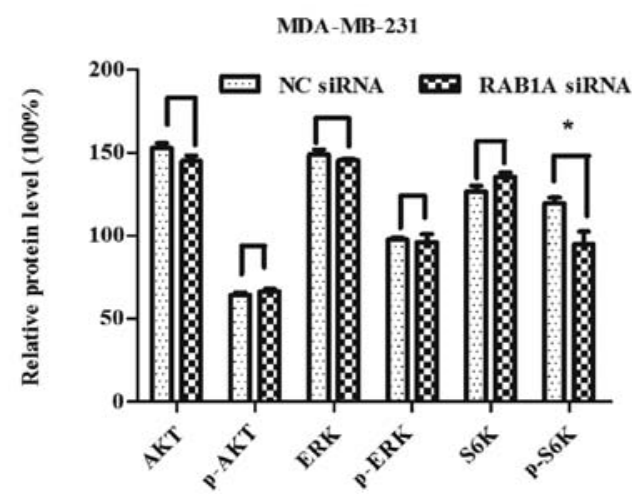

B

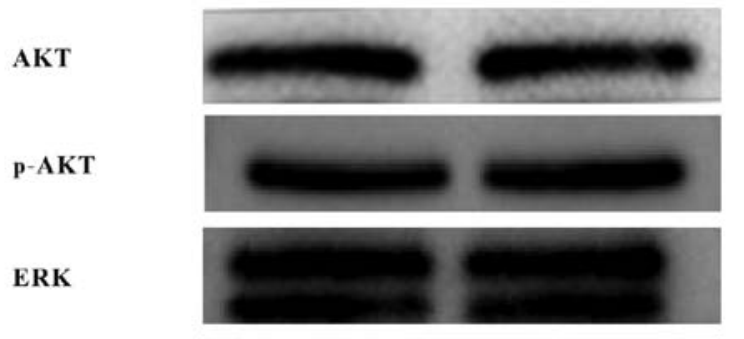

p-ERK

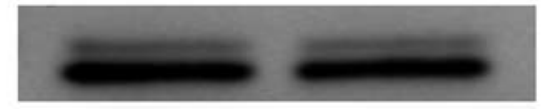

S6K

P70S6K

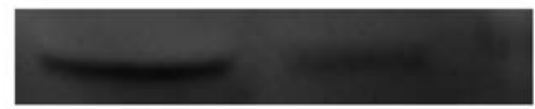

Tubulin

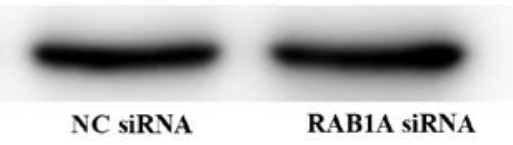

D

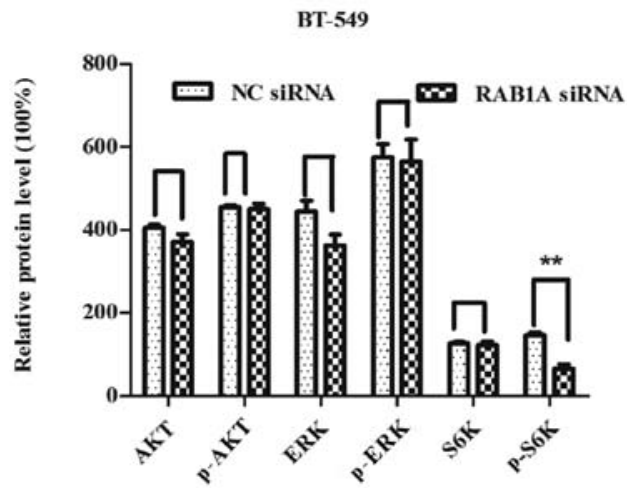

Figure 3. RAB1A siRNA is involved in restraining the mTORC1 pathway. In (A and C) MDA-MB-231 and (B and D) BT-549 cells, RAB1A knockdown did not decrease AKT, p-AKT, ERK, p-ERK, P70S6K protein levels, while inhibited the expression of p-P70S6K, the effector of mTORC1. The graph shows the mean \pm SEM of AKT, p-AKT, ERK, p-ERK, P70S6K, p-P70S6K protein levels related to their loading control. Quantitative analysis was conducted by measuring the integrated density value of bands. Blots are representative of results from 3 experiments. ${ }^{*} \mathrm{P}<0.05,{ }^{* * *} \mathrm{P}<0.01$ (two-tailed $\mathrm{t}$-test).

RABIA siRNA inhibits the expression of mTORC1 effector $p$-P70S6K. To verify the relationship between RAB1A and S6K. RAB1A expression was suppressed by RAB1A siRNA and the expression of S6K was detected by western blotting. According to Fig. 3A and $\mathrm{B}$, following the downregulation of RAB1A in protein level, protein expression of p-P70S6K was decreased in breast cancer cells, while the expression of p-ERK or p-AKT had no change. All the results above indicate the vital role of RAB1A in regulation of activation of $\mathrm{S} 6 \mathrm{~K}$. The integrated density values of bands were as shown in Fig. $3 \mathrm{C}$ and $\mathrm{D}(\mathrm{P}<0.05, \mathrm{P}<0.01)$.

Proliferation ability of breast cancer cells is suppressed by the $R A B 1 A$ siRNA. To investigate the impact of siRAB1A on the proliferation ability of breast cancer cells, both MDA-MB-231 and BT-549 cells transfected with RAB1A siRNA or non-specific NC were used in the MTT assay. Transfections were performed as described above. Absorbance of the two groups was recorded at 24, 48, 72, 96 and $120 \mathrm{~h}$. Briefly, inhibition rate was calculated as: inhibition rate $(\%)=(\mathrm{OD}$ value of the control group - OD value of experimental group)/OD value of the control group $x 100 \%$. A great suppression of cell viability at $120 \mathrm{~h}$ in RAB1A siRNA transfected cells was detected compared with the control group, both in MDA-MB-231 cells (inhibition rate $=14.78 \pm 6.54 \%$, Fig. $4 \mathrm{~A} ; \mathrm{P}<0.01$ ) and BT-549 cells (inhibition rate $=25.06 \pm 9.20 \%$, Fig. $4 \mathrm{~B} ; \mathrm{P}<0.001$ ). These results indicated RAB1A depletion suppressed cellular proliferation.

Colony formation was inhibited by the RABIA siRNA in breast cancer cells. As shown in Fig. 5A and B, RAB1A siRNA transfected cells exhibited fewer colonies than NC transfected cells in colony formation assays. The colony formation rate in RAB1A siRNA-transfected MDA-MB-231 was significantly decreased compared with the NC transfected cells (Fig. 5C; $\mathrm{P}<0.01$ ). The same tendency was shown in 
A

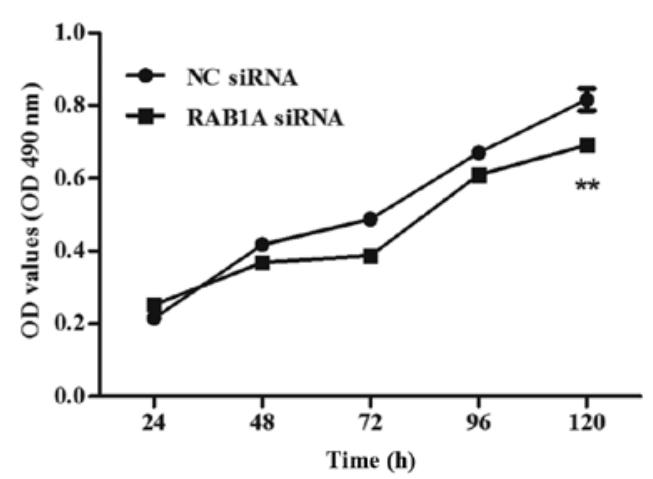

B

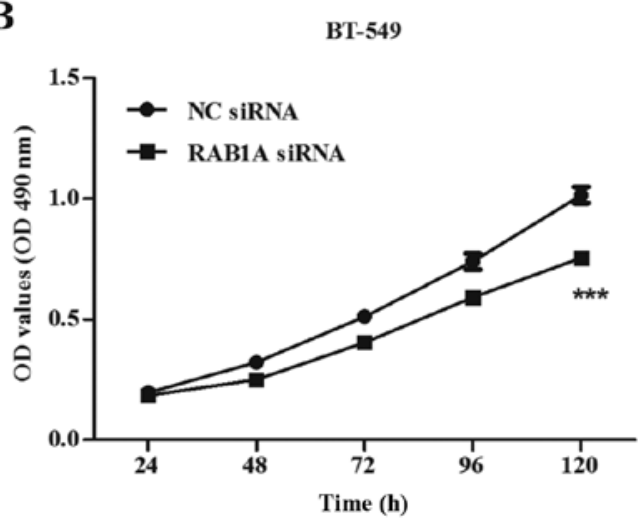

Figure 4. Knockdown of RAB1A suppresses proliferation of cells. (A) Transfection of RAB1A siRNA inhibited MDA-MB-231 cells growth. ${ }^{* *} \mathrm{P}<0.01$ vs. the control by two-tailed t-test. (B) In BT-549, the same trend was seen. ${ }^{* * * *} \mathrm{P}<0.001$ vs. the control by two-tailed t-test. Graph represents OD $490 \pm$ SEM.

A

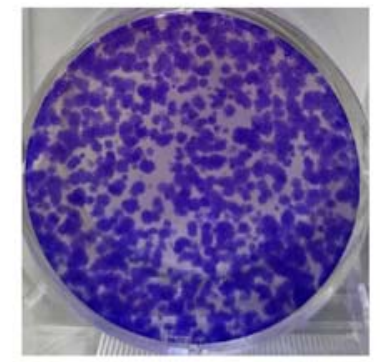

NC SIRNA
MDA-MB-231

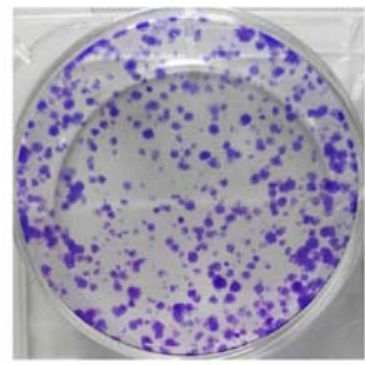

RAB1A siRNA
C

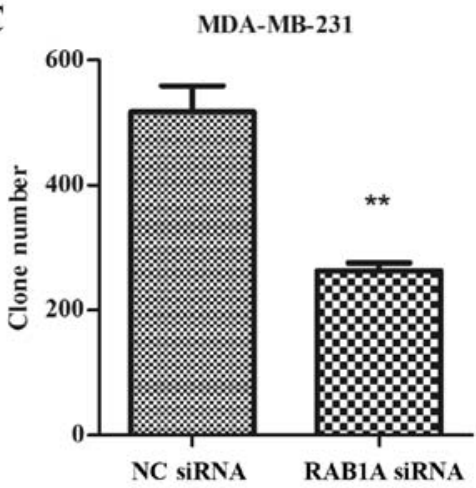

D

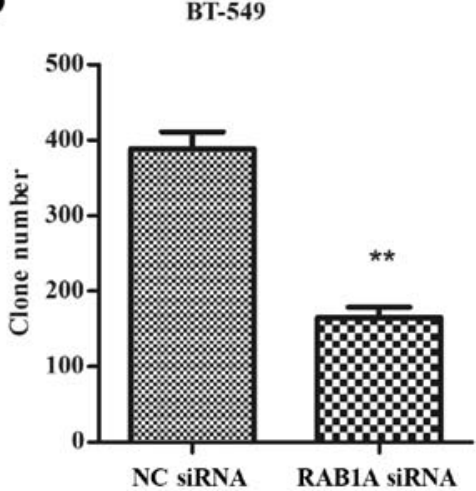

Figure 5. Downregulation of RAB1A inhibits colony formation ability of breast cancer cells. Representative images of crystal violet stained colonies in (A) MDA-MB-231 and (B) BT-549 cells transfected with RAB1A siRNA or NC siRNA are shown. Cells transfected with RAB1A siRNA exhibited fewer colonies than the NC group in $\left(\mathrm{C},{ }^{* *} \mathrm{P}<0.01\right)$ MDA-MB-231 and $\left(\mathrm{D},{ }^{* *} \mathrm{P}<0.01\right) \mathrm{BT}-549$ cells. Graphs show mean $\pm \mathrm{SEM}$ of numbers of colonies (two-tailed t-test). Results are representative of two similar experiments.

BT-549 cells (Fig. 5D; P<0.01). Taken together, these data indicated that depletion of RAB1A inhibited clonogenesis of breast cancer cells.

RABIA siRNA reduces cell invasion and metastasis in vitro. To evaluate the effect of RAB1A siRNA on breast cancer metastasis, two aggressive breast cancer cell lines MDA-MB-231 and BT-549 were transiently transfected with RAB1A siRNA or NC control. The invasion and migration of cells were measured by Transwell assays and wound healing assays, respectively. All the results revealed that the number of MDA-MB-231 or BT-549 cells penetrated to the lower membrane was significantly decreased at $16 \mathrm{~h}$ after RAB1A siRNA transfection compared to the control group (Fig. 6). Notably, as shown in Fig. 7, RAB1A siRNA but not NC siRNA markedly decrease the migratory property of these breast cancer cells.

RAB1A depletion suppresses EMT in breast cancer cells. Next, we explored the role of RAB1A siRNA in breast cancer EMT. Western blotting were carried out. Fig. 8A and C shows, the expression of E-cadherin protein, an epithelial 


\section{A}

MDA-MB-231
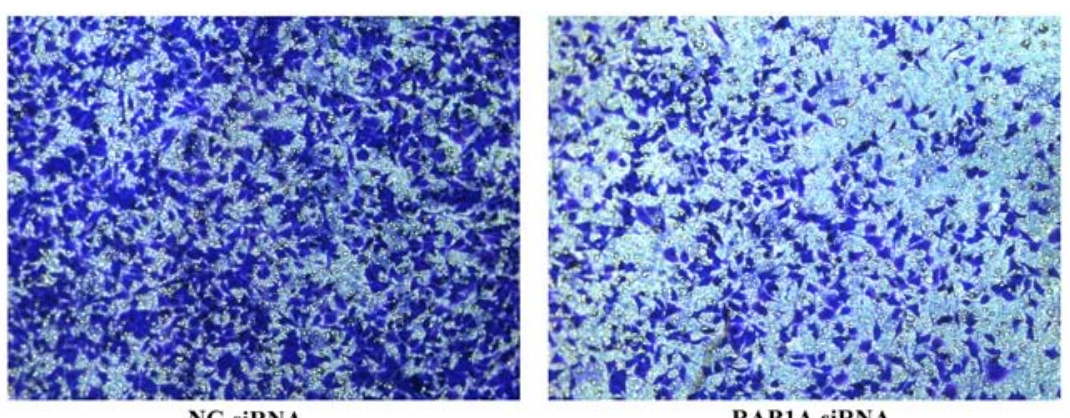

B

\section{BT-549}
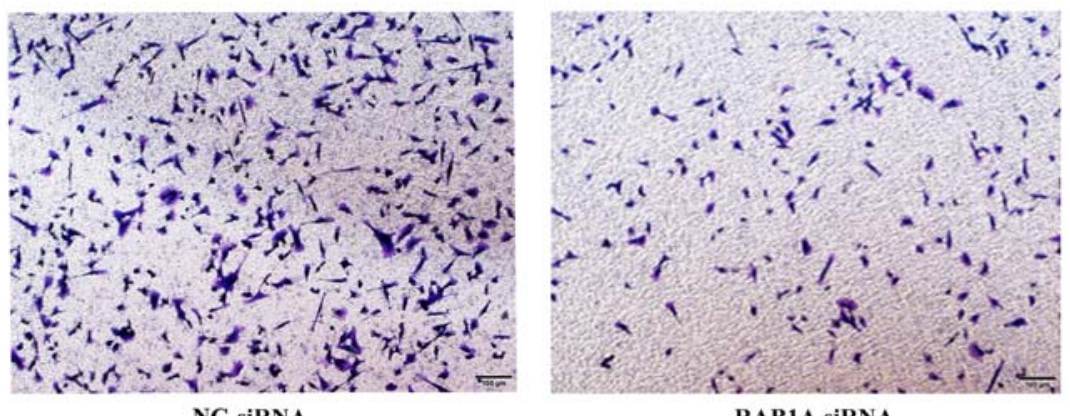

NC siRNA

RAB1A siRNA

Figure 6. Knockdown of RAB1A suppresses breast cancer cell invasion. Transwell assays were performed to detect motility changes in (A) MDA-MB-231 and (B) BT-549 cells which were transfected with RAB1A siRNA or NC siRNA. Representative images of crystal violet-stained invasive cells on the membrane are shown. Results are representative of two similar experiments.

A
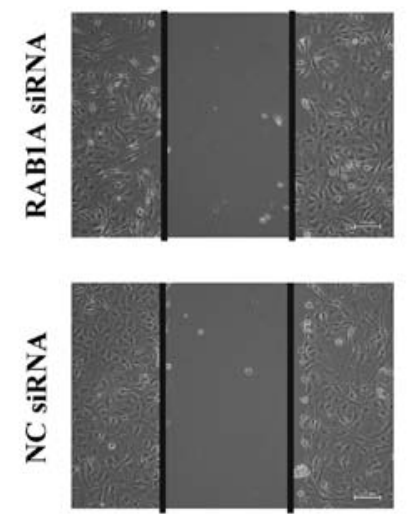

$\mathbf{0 h}$

B

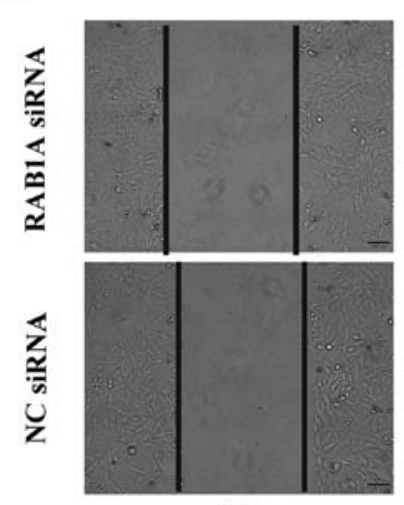

$\mathbf{0 ~ h}$
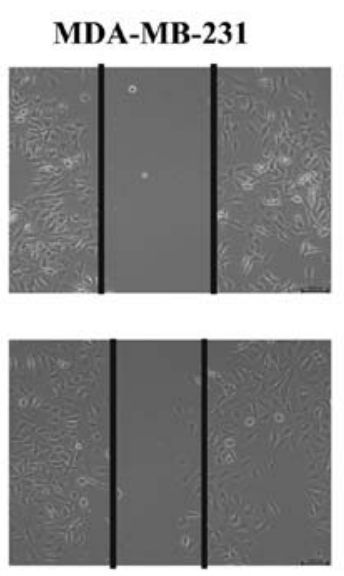

$12 \mathrm{~h}$

BT-549
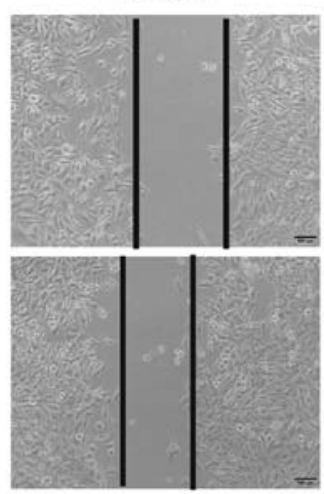

$12 \mathrm{~h}$
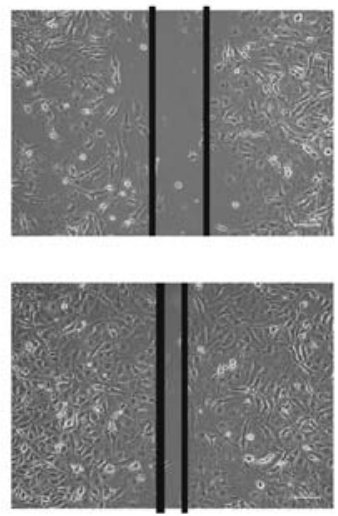

$24 \mathrm{~h}$
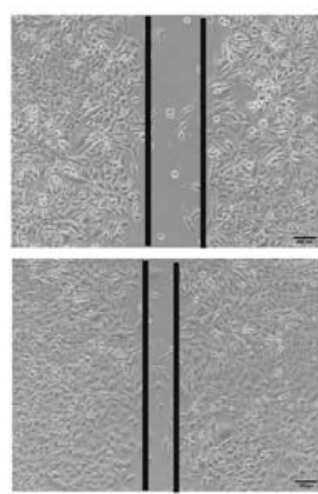

$24 \mathrm{~h}$

Figure 7. The loss of RAB1A inhibits cell migration. In (A) MDA-MB-231 and (B) BT-549 cell lines, in comparison to cells treated with NC siRNA, cells treated with RAB1A siRNA showed a significant decrease in migration as assessed by wound healing assay. 
A

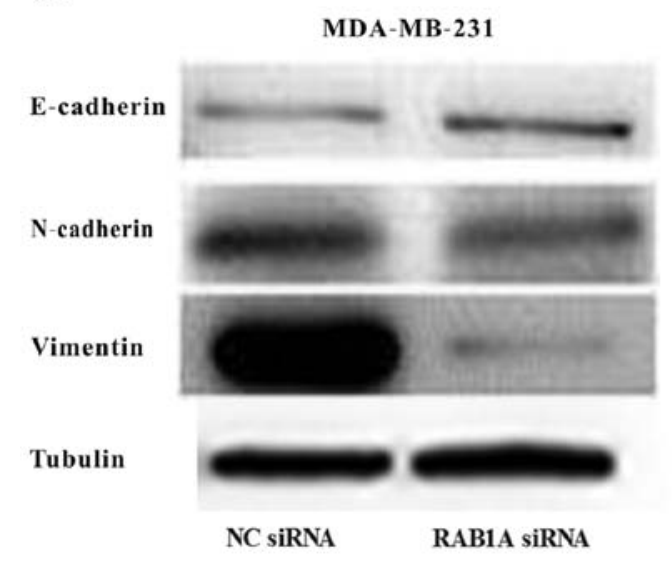

C

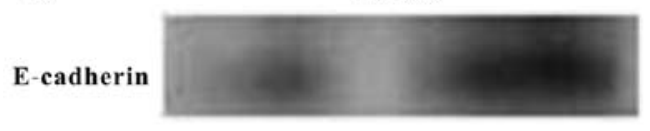

$\mathrm{N}$-cadherin

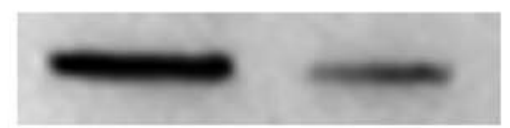

Vimentin

Tubulin

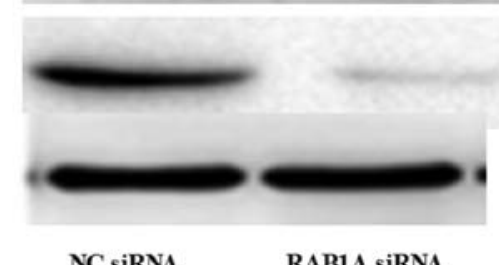

B

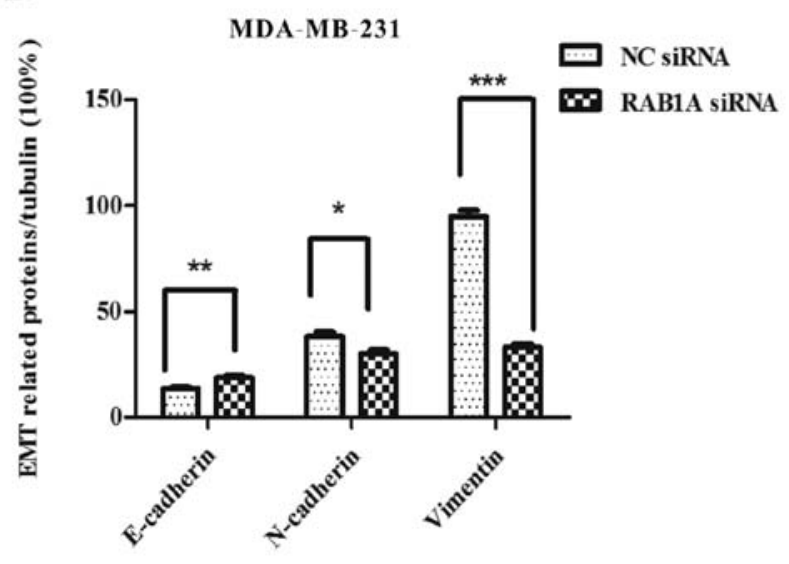

D

BT-549

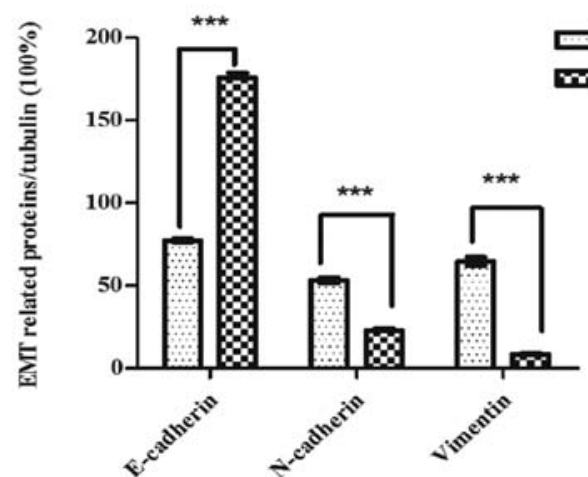

Figure 8. RAB1A siRNA represses EMT in breast cancer. Both in (A and B) MDA-MB-231 and (C and D) BT-549 cells, E-cadherin protein was significantly upregulated in the presence of RAB1A siRNA while the expression of N-cadherin and vimentin reduced apparently. The graph shows the mean \pm SEM of E-cadherin, $\mathrm{N}$-cadherin and vimentin protein levels related to their loading control. Quantitative analysis was conducted by measuring the integrated density value of bands. Data were obtained from three independent experiments. ${ }^{*} \mathrm{P}<0.05,{ }^{* * *} \mathrm{P}<0.01,{ }^{* * * *} \mathrm{P}<0.001$ (two-tailed t-test). EMT, epithelial-mesenchymal transition.

cell marker, was increased in RAB1A siRNA transfected MDA-MB-231 and BT-549 cells while the mesenchymal markers $\mathrm{N}$-cadherin and vimentin were decreased. The integrated density values of bands were measured and shown (Fig. 8B and $\mathrm{D} ; \mathrm{P}<0.05, \mathrm{P}<0.01, \mathrm{P}<0.001$ ). Taken together, these results indicated that $\mathrm{RAB} 1 \mathrm{~A}$ depletion in breast cancer cells may be responsible for the EMT suppression and cell migration suppression.

\section{Discussion}

As the leading cause of cancer-related death among women worldwide, breast cancer have a severe influence on the living standard of the patients. During the investigation of the potential role in breast cancer, RAB1A siRNA was transfected in the MDA-MB-231 and BT-549 cells. Cell invasion and proliferation abilities were significantly abolished by depletion of RAB1A according to the Transwell and MTT assay, respectively. Moreover, our data showed that the downregulated expression of RAB1A increased the epithelial cell marker E-cadherin in breast cancer cells, while the mesenchymal cell markers, $\mathrm{N}$-cadherin and vimentin, were decreased (17). The loss of E-cadherin mediated adherens junction was the first step in EMT and plays a vital role in cells invasiveness and distance metastasis (18). All the results indicate EMT suppression mediated by exogenous silencing of RAB1A contributing to the inhibition of breast cancer metastasis.

mTOR pathway as an important regulator in malignant tumor process consists of two complexes, mTORC1 and mTORC2. As an important effector of mTORC1, p-P70S6K plays a vital role in regulating cell growth, invasion, and lymph node metastasis in colorectal cancer (19). In this experiment, downregulated expression of RAB1A by transfection with RAB1A siRNA suppressed the expression of p-P70S6K, rather than the p-AKT and p-ERK, which revealed a close connection between RAB1A and p-P70S6K and the reasons for the suppression of proliferation and metastasis by exogenous silencing of RAB1A gene in breast cancer.

Several groups have already investigated the function of RAB1A in colorectal cancer and what we found in this study was in accordance with previous reports that RAB1A depletion was involved in the suppression of mTORC1 pathway and it acts in oncogenesis of breast cancer proliferation, growth and metastasis. Our results may help the finding of new strategies for the treatment of breast cancer. 
A number of limitations should be noted in this study. Clinical specimens were not used. The relationship between RAB1A expression and patient survival was not identified. Therefore, further studies are needed.

\section{Acknowledgements}

This study was supported by the National Natural Sciences Foundation of China for the project 81272240.

\section{References}

1. Chen W, Zheng R, Baade PD, Zhang S, Zeng H, Bray F, Jemal A, Yu XQ and He J: Cancer statistics in China, 2015. CA Cancer J Clin 66: 115-132, 2016.

2. Jemal A, Bray F, Center MM, Ferlay J, Ward E and Forman D Global cancer statistics. CA Cancer J Clin 61: 69-90, 2011.

3. Torre LA, Bray F, Siegel RL, Ferlay J, Lortet-Tieulent J and Jemal A: Global cancer statistics, 2012. CA Cancer J Clin 65: 87-108, 2015.

4. Schöppner P, Csaba G, Braun T, Daake M, Richter B, Lange OF, Zacharias M, Zimmer R and Haslbeck M: Regulatory implications of non-trivial splicing: isoform 3 of Rab1A shows enhanced basal activity and is not controlled by accessory proteins. J Mol Biol 428: 1544-1557, 2016.

5. Allan BB, Moyer BD and Balch WE: Rab1 recruitment of p115 into a cis-SNARE complex: programming budding COPII vesicles for fusion. Science 289: 444-448, 2000.

6. Satoh A, Wang Y, Malsam J, Beard MB and Warren G: Golgin-84 is a rab1 binding partner involved in Golgi structure. Traffic 4: $153-161,2003$.

7. Coune PG, Bensadoun JC, Aebischer P and Schneider BL: Rab1A over-expression prevents Golgi apparatus fragmentation and partially corrects motor deficits in an alpha-synuclein based rat model of Parkinson's disease. J Parkinsons Dis 1: 373-387, 2011

8. Park JS, Heo JS, Chang HS, Choi IS, Kim MK, Lee JU, Park BL, Shin HD and Park CS: Association analysis of member RAS oncogene family gene polymorphisms with aspirin intolerance in asthmatic patients. DNA Cell Biol 33: 155-161, 2014.

9. Wu G, Yussman MG, Barrett TJ, Hahn HS, Osinska H, Hilliard GM, Wang X, Toyokawa T, Yatani A, Lynch RA, et al: Increased myocardial Rab GTPase expression: a consequence and cause of cardiomyopathy. Circ Res 89: 1130-1137, 2001.
10. Shimada K, Uzawa K, Kato M, Endo Y, Shiiba M, Bukawa H, Yokoe H, Seki N and Tanzawa H: Aberrant expression of RAB1A in human tongue cancer. Br J Cancer 92: 1915-1921, 2005.

11. Yang Y, Hou N, Wang X, Wang L, Chang S, He K, Zhao Z, Zhao X, Song T and Huang C: miR-15b-5p induces endoplasmic reticulum stress and apoptosis in human hepatocellular carcinoma, both in vitro and in vivo, by suppressing Rab1A. Oncotarget 6: 16227-16238, 2015.

12. Wang $\mathrm{X}$, Chu Y, Wang W and Yuan W: mTORC signaling in hematopoiesis. Int J Hematol 103: 510-518, 2016.

13. Thomas JD, Zhang YJ, Wei YH, Cho JH, Morris LE, Wang HY and Zheng XF: Rab1A is an mTORC1 activator and a colorectal oncogene. Cancer Cell 26: 754-769, 2014.

14. Sun T, Wang X, He HH, Sweeney CJ, Liu SX, Brown M, Balk S, Lee GS and Kantoff PW: miR-221 promotes the development of androgen independence in prostate cancer cells via downregulation of HECTD2 and RAB1A. Oncogene 33: 2790-2800, 2014.

15. Xu BH, Li XX, Yang Y, Zhang MY, Rao HL, Wang HY and Zheng XF: Aberrant amino acid signaling promotes growth and metastasis of hepatocellular carcinomas through Rab1A-dependent activation of mTORC1 by Rab1A. Oncotarget 6: 20813-20828, 2015

16. Gulhati P, Bowen KA, Liu J, Stevens PD, Rychahou PG, Chen M, Lee EY, Weiss HL, O'Connor KL, Gao T, et al: mTORC1 and mTORC2 regulate EMT, motility, and metastasis of colorectal cancer via RhoA and Racl signaling pathways. Cancer Res 71: 3246-3256, 2011

17. Makki J, Myint O, Wynn AA, Samsudin AT and John DV: Expression distribution of cancer stem cells, epithelial to mesenchymal transition, and telomerase activity in breast cancer and their association with clinicopathologic characteristics. Clin Med Insights Pathol 8: 1-16, 2015.

18. Zheng K, Zhou X, Yu J, Li Q, Wang H, Li M, Shao Z, Zhang F, Luo Y, Shen Z, et al: Epigenetic silencing of miR-490-3p promotes development of an aggressive colorectal cancer phenotype through activation of the $\mathrm{Wnt} / \beta$-catenin signaling pathway. Cancer Lett 376: 178-187, 2016.

19. Lu Q, Wang J, Yu G, Guo T, Hu C and Ren P: Expression and clinical significance of mammalian target of rapamycin/P70 ribosomal protein S6 kinase signaling pathway in human colorectal carcinoma tissue. Oncol Lett 10: 277-282, 2015. 